\title{
Analyse géostatistique des répartitions horizontales printanières de la biomasse zooplanctonique et des variables physico-chimiques dans un petit lac Geostatistical analysis of horizontal distributions of zooplankton biomass and environmental variables in a small lake during the spring season
}

\author{
Nadine Angeli, Daniel Gerdeaux et Jean Guillard
}

Volume 19, numéro 4, 2006

URI : https://id.erudit.org/iderudit/014416ar

DOI : https://doi.org/10.7202/014416ar

\section{Aller au sommaire du numéro}

\section{Éditeur(s)}

Université du Québec - INRS-Eau, Terre et Environnement (INRS-ETE)

\section{ISSN}

1718-8598 (numérique)

Découvrir la revue

\section{Citer cet article}

Angeli, N., Gerdeaux, D. \& Guillard, J. (2006). Analyse géostatistique des répartitions horizontales printanières de la biomasse zooplanctonique et des variables physico-chimiques dans un petit lac. Revue des sciences de l'eau / Journal of Water Science, 19(4), 285-294. https://doi.org/10.7202/014416ar

\section{Résumé de l'article}

À partir d'un échantillonnage intensif du lac de Nantua, nous avons déterminé à quelles échelles les structures spatiales de la biocénose et de son environnement sont le plus fortement exprimées à l'aide de techniques géostatistiques. Ces résultats obtenus au printemps portent sur des descripteurs globaux (biomasse totale, moyennes déduites des profils verticaux enregistrés par sonde multiparamètre). Les modèles de variogrammes ajustés aux données montrent qu'une forte part de la variance totale s'exprimait à grande échelle pour le zooplancton total, à très petite échelle, au contraire, pour les descripteurs reflétant l'activité métabolique lacustre. La liaison des différentes variables explorées avec la température variait en outre fortement avec l'augmentation de la distance inter-stations, confortant l'hypothèse que des facteurs structurants distincts se succédaient sur la plage d'échelles considérée. Cette étude fondée sur des variables globales souligne la difficulté d'extrapoler les liaisons entre variables ou processus à d'autres échelles que celle(s) observée(s). 


\title{
ANALYSE GÉOSTATISTIQUE DES RÉPARTITIONS HORIZONTALES PRINTANIÈRES DE LA BIOMASSE ZOOPLANCTONIQUE ET DES VARIABLES PHYSICO- CHIMIQUES DANS UN PETIT LAC
}

\author{
Geostatistical analysis of horizontal distributions of zooplankton biomass and environmental variables in a small \\ lake during the spring season
}

\author{
Nadine Angeli, Daniel Gerdeaux et Jean Guillard* \\ I.N.R.A., Station d'Hydrobiologie Lacustre, UMR CARRTEL, B.P.511F, 74203 Thonon Cedex
}

Reçu le 5 juillet 2005, accepté le 17 février 2006

\section{RÉSUMÉ}

À partir d'un échantillonnage intensif du lac de Nantua, nous avons déterminé à quelles échelles les structures spatiales de la biocénose et de son environnement sont le plus fortement exprimées à l'aide de techniques géostatistiques. Ces résultats obtenus au printemps portent sur des descripteurs globaux (biomasse totale, moyennes déduites des profils verticaux enregistrés par sonde multiparamètre). Les modèles de variogrammes ajustés aux données montrent qu'une forte part de la variance totale s'exprimait à grande échelle pour le zooplancton total, à très petite échelle, au contraire, pour les descripteurs reflétant l'activité métabolique lacustre. La liaison des différentes variables explorées avec la température variait en outre fortement avec l'augmentation de la distance interstations, confortant l'hypothèse que des facteurs structurants distincts se succédaient sur la plage d'échelles considérée. Cette étude fondée sur des variables globales souligne la difficulté d'extrapoler les liaisons entre variables ou processus à d'autres échelles que celle(s) observée(s).

Mots clefs : structures spatiales, plancton lacustre, géostatistique, coefficient de codispersion.

\section{ABSTRACT}

On the basis of a dense sampling survey, and for a given site and a given season (Lake Nantua; mid-spring), we assessed on which scales the spatial patterns of the biocenosis and its environment were most strongly expressed. These preliminary results focused on global descriptors (total biomass per surface unit, means computed from vertical multiprobe recordings). The theoretical variograms fitted to the data showed that most of the total variance was expressed on a broad scale for total zooplankton biomass. In contrast, the variance of descriptors reflecting lake metabolic processes was expressed on very short scales. For most of the explored descriptors, their relations with temperature also varied dramatically on a spatial scale, supporting the hypothesis that distinct structural factors prevail at different scales. The practical implications of these multiscale characteristics are discussed.

Key words: spatial patterns, lacustrine plankton, geostatistics, co-dispersion coefficient. 


\section{INTRODUCTION}

Bien que l'importance et la prédictibilité de l'hétérogénéité des organismes pélagiques soient déterminantes pour le choix des stratégies d'échantillonnage (DUTILLEUL, 1993); DUTILLEUL et LEGENDRE, 1993; LEGENDRE, 1993; LEGENDRE et FORTIN, 1989), et que des études de plus en plus nombreuses tentent d'intégrer cette caractéristique fonctionnelle multiscalaire de l'écosystème, la grande majorité des suivis effectués sur les lacs se limite à une perception très locale du système : la station dite de référence. Les structures spatiales du zooplancton lacustre ont le plus souvent été étudiées à partir de profils verticaux ou temporels à une station donnée (BÜRGI et al., 1993; GELLER et al., 1992; HANAZATO, 1992; SOMMER, 1989; WEIDER et STICH, 1992). Plusieurs études tiennent compte de la répartition horizontale du zooplancton lacustre généralement sur un ou quelques transects (GLIWICZ et RYKOWSKA, 1992; JOHANNSSON et al., 1991; PINEL-ALLOUL et PONT, 1991; URABE, 1990), très exceptionnellement sur la totalité d'un lac (AVOIS et al., 2000; KALIKHMAN et al., 1992; LACROIX et LESCHER-MOUTOUÉ, 1995; MASSON et al., 2004; PATALAS et SALKI, 1992; VILJANEN et KARJALAINEN, 1993). Ce constat s'explique d'une part par le caractère onéreux des séries denses et, d'autre part, par l'inadéquation des équipements conventionnels de récolte vis-à-vis des variations des structures spatiales sur l'axe horizontal (DIRNBERG et THRELKELD, 1986; HART, 1978). Pour contourner les problèmes inhérents à la lenteur des engins de récolte, nous avons ciblé cette étude sur le lac de Nantua, petit lac sub-alpin. Ces échantillonnages qui ont été effectués en début de période de réchauffement des eaux (mai), visent à préciser :

1) l'ordre de grandeur des variations impliquées;

2) les échelles de plus forte variabilité, déterminant l'effort d'échantillonnage minimal à consentir pour une étude systématique des facteurs structurants.

Nous examinerons donc ici, pour un ensemble de descripteurs environnementaux et biologiques, de quelle façon évoluent avec l'échelle d'observation (distance inter-station) la variance de ces descripteurs et leur liaison avec la température, variable indépendante et variable la plus structurante.

\section{CARACTÉRISTIQUES DU SITE}

Le lac de Nantua (superficie: 1,4 $\mathrm{km}^{2}$; profondeur maximale: $42 \mathrm{~m}$ ) (Figure 1) est situé en aval de la ville homonyme, dans une vallée encaissée et exposée à un microclimat pluvieux. Le fonctionnement et la structure de cet écosystème sont fortement conditionnés par la dominance des oscillaires (Planktothrix rubescens) et par leur mode d'occupation de l'espace (FEUILLADE, 1985; FEUILLADE et DRUART, 1994). Le zooplancton, dont la biomasse est comparativement très faible, compte de nombreux ciliés et rotifères, quelques copépodes et entomostracés, et les larves d'un Chaoboridae.

\section{MÉTHODOLOGIE}

\section{1 Échantillonnage.}

Les prélèvements, effectués le 2 mai 1990 par un temps calme et un ciel clair, sont réalisés sur un pas d'échantillonnage irrégulier, dû à des repérages à vue, un positionnement par des amers et un relevé au compas, sans ancrage. Le lac est couvert en moins d'une douzaine d'heures. Les récoltes planctoniques sont issues de traits verticaux ( $0-20 \mathrm{~m}$ ou 0 -fond quand le fond est inférieur à $20 \mathrm{~m}$ ) au filet biconique (maille de $50 \mu \mathrm{m}$, ayant un bon rendement de filtration assuré par un rapport surface filtrante/ouverture de 1/20). Les descripteurs physico-chimique ont été enregistrés à l'aide d'une sonde multiparamètre (Sonde M.E., Standard-Eco-probe version 11) dans la strate 0-13 m (Figure1) pour 54 stations sur 71 (correspondant à la mémoire interne de la sonde).

\subsection{Traitement des échantillons}

Échantillons biologiques: les fixations ont été faites sur le terrain (formol à $3 \%$ ). Compte tenu de la prépondérance des oscillaires dans ce milieu, l'énorme masse de trichomes recueillis au filet fin peut être considérée comme un descripteur de la biomasse totale du phytoplancton. La masse d'oscillaires a été évaluée par pesée sur microbalance après concentration sur filtre d'une fraction d'échantillon brut et $24 \mathrm{~h}$ de séchage à $60^{\circ} \mathrm{C}$. Nous avons procédé de même pour le zooplancton métazoaire, après post-fixation des échantillons (formol $10 \%$ ) et extraction des animaux du feutrage d'oscillaires. Cette extraction a été faite sur de larges tamis à sédiments ayant la même maille que l'engin de récolte. On obtient par succion une élimination efficace des trichomes en agitant verticalement le tamis à la surface d'une masse d'eau renouvelée. Les résultats sont exprimés en densité de surface dans un souci de cohérence avec d'autres travaux menés conjointement (PINEL-ALLOUl et al., 1999) et en relation avec l'énergie lumineuse calculée en fonction de la surface du lac.

Enregistrements multiparamètres: Les grandeurs analysées sont les valeurs moyennes du segment 2 - $13 \mathrm{~m}$ de la colonne d'eau, la couche superficielle étant éliminée afin d'éviter les variations journalières dues au réchauffement. Les variables environnementales sont la température, le $\mathrm{pH}$, la turbidité (FTU : Formazin Turbidity Units) exprimée comme un pourcentage de transparence par rapport à l'air, le pourcentage 


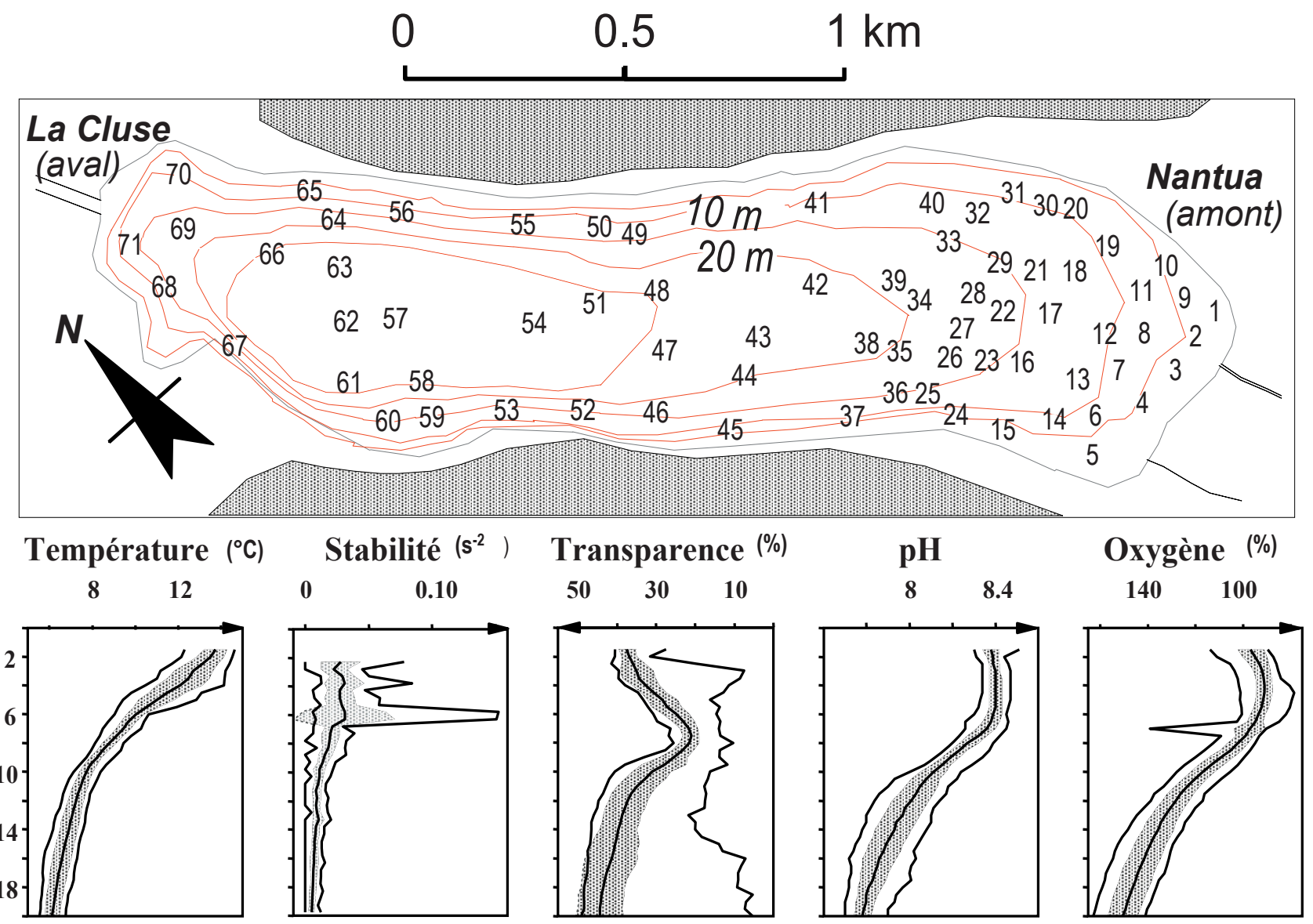

Figure 1 Bathymétrie, localisations des stations et principaux paramètres physico-chimiques (moyennes, écart-types (grisé) et amplitudes maximales de variation).

Bathymetry, locations of the stations and main physicochemical parameters (averages, standard deviation (greyed) and maximum amplitudes of variation).

d'oxygène dissous, ainsi qu'un descripteur de stabilité verticale : la fréquence de Brünt-Vâisälä $\left(\mathrm{N}^{2}\right)$ (LEMMIN, 1995), déduite des profils thermiques à partir d'une des approximations paraboliques utilisées dans le programme MELIMEX (IMBODEN et al., 1979). Cette méthode permet d'estimer l'intensité des mélanges à chaque station, caractérisant ainsi la stabilité verticale (REYNOLDS, 1984).

Pour $\varrho(T) \approx 1000-7^{*} 10^{-3}(T-4)^{2}$, l'équation de BrüntVäisälä peut s'écrire :

$$
\mathrm{N}^{2}=\frac{\mathrm{g}}{\rho} * \frac{\mathrm{d} \rho}{\mathrm{d} z} \approx \frac{9.81}{1000} * \frac{\rho\left(\mathrm{T}_{\mathrm{z} 2}\right)-\rho\left(\mathrm{T}_{\mathrm{z} 1}\right)}{\mathrm{Z}_{2}-\mathrm{Z}_{1}}
$$

avec : $\mathrm{N}^{2}\left(\right.$ en cycles s $\left.\mathrm{s}^{-2}\right) ; \mathrm{g}$ = accélération de la gravité $\left(\right.$ en $\left.\mathrm{m} \mathrm{s}^{-2}\right)$, $\varrho=$ densité de l'eau pure $\left(\mathrm{en} \mathrm{kg} \mathrm{m}^{-3}\right) ; \mathrm{T}=$ température $\left(\mathrm{en}^{\circ} \mathrm{C}\right)$; $\mathrm{Z}_{1}$ et $\mathrm{Z}_{2}$ : profondeurs discrètes (en $\mathrm{m}$ et distantes de $0,5 \mathrm{~m}$ dans les calculs).

\subsection{Traitements géostatistiques.}

Démarche pratique: L'analyse se fonde sur la cartographie des données standardisées, dont l'étendue a arbitrairement été amenée à 0-100 par la transformation $\left(\mathrm{x}-\mathrm{x}_{\min }\right) /\left(\mathrm{x}_{\max }-\mathrm{x}_{\min }\right) * 100$, et sur le variogramme direct ou croisé avec la température. Compte tenu de la forme allongée du lac (faible nombre de couples de valeurs dans certaines directions), c'est le variogramme moyen qui a été calculé. Les classes de distances retenues suivent une progression arithmétique de raison $80 \mathrm{~m}$ (tolérance $40 \mathrm{~m}$ ). Ces fonctions structurelles ont été établies respectivement, pour les cas uniet bi-variés, avec les logiciels EVA (PETITGAS et LAFONT, 1997) et GEOPACK (YATES et al., 1989). L'ajustement aux données d'un variogramme théorique, choisi parmi les modèles les plus couramment utilisés en géostatistique (puissance/linéaire, sphérique ou gaussien) (JOURNEL et HUIJBREGTS, 1978) a été fait de visu. On peut trouver dans ARMSTRONG et al. (1992), JOURNEL et HUIJBREGTS (1978), et MATHERON (1971), les éléments relatifs aux calculs et aux modèles utilisés.

Quantification de la liaison spatiale entre deux variables: Pour tout couple de variables $Z_{\mathrm{i}}$ et $Z_{\mathrm{J}}$, le coefficient de codispersion spatiale $\mathrm{r}_{\mathrm{ij}}(\mathrm{h})=\gamma_{\mathrm{ij}}(\mathrm{h}) / \sqrt{\gamma_{\mathrm{ii}}(\mathrm{h}) \cdot \gamma_{\mathrm{ij}}(\mathrm{h})}$ (WACKERNAGEL, 1988) ou rapport entre le variogramme croisé et la racine carrée du produit des variogrammes directs, 
décrit la façon dont la corrélation entre les incréments spatiaux de $Z_{i}$ et $Z_{i}$, varie en fonction de l'échelle considérée. Sous l'hypothèse de stationnarité des accroissements et du comportement asymptotique des variogrammes, la limite de $\mathrm{r}_{\mathrm{ij}}(\mathrm{h})$ quand $|\mathrm{h}| \rightarrow \infty$ est le coefficient de corrélation linéaire (WACKERNAGEL, 1988). Si ce coefficient demeure constant pour toutes les valeurs de $\mathrm{h}$, cela signifie que la liaison entre les deux variables ne se modifie pas avec l'échelle spatiale; on mesure ainsi comment la liaison entre deux paramètres varie en fonction des distances (GOOVAERTS, 1992).

\section{RÉSULTATS}

Lors de la campagne, la stratification des masses d'eau a commencé à s'installer depuis deux mois. La strate épilimnique, épaisse de 9 à $10 \mathrm{~m}$ environ, est cependant encore assez fraîche (on passe en moyenne de $13,5^{\circ} \mathrm{C}$ à $7,8^{\circ} \mathrm{C}$ entre $1 \mathrm{~m}$ et $9 \mathrm{~m}$ ) et les gradients verticaux sont encore peu contrastés.

\section{1 Étendue des séries}

On constate (Tableau 1) que les amplitudes et coefficients de variations relatifs aux biomasses (rapports $\mathrm{max} / \mathrm{min}$ respectivement de 2,7 et 17 pour le phytoplancton et le zooplancton; coefficient de variation de $23 \%$ et $61 \%$ ) contrastent avec la très faible variabilité horizontale des descripteurs environnementaux (CV de 9 à $10 \%$ et rapports $\max / \min <2,4$ pour la stabilité thermique et la transparence; CV compris entre 0,4 et 2,1\%, pour la température, le $\mathrm{pH}$ et l'oxygène dissous, rapports $\max / \mathrm{min}<1,2$ pour le $\mathrm{pH}$ et $\mathrm{O}_{2}$ ).

\subsection{Distribution spatiale et gradients:}

La cartographie suggère l'existence d'une structuration spatiale pour toutes les variables (Figure 2), y compris celles qui présentent une très faible étendue. Les moyennes pondérées se répartissent pour la température selon un gradient conduisant à une structure en croissant. Les faibles valeurs sont relevées aux deux cinquièmes de la longueur du lac (si l'on se positionne depuis la rive N.-O.) avec les minima du côté de la rive S.-O. et les valeurs intermédiaires se répartissant en auréole autour de cette région; alors que les valeurs excédant $80 \%$ de l'étendue de cette variable sont toutes localisées dans le quart S.-E. de la cuvette (du côté de Nantua et dans une région de plus faible profondeur moyenne du bassin). Les gradients de transparence et stabilité présentent de fortes analogies avec ce schéma de répartition, sauf dans la partie N.-O. du lac où de faibles valeurs de stabilité sont associées à des valeurs intermédiaires de température. Pour l'oxygène dissous, la biomasse zooplanctonique et les cyanobactéries, le gradient est plus longitudinal et de sens opposé à celui de la température. Le $\mathrm{pH}$ présente des valeurs élevées au centre du lac et faibles aux deux extrémités, en opposition avec les structures spatiales des autres paramètres.

Tableau 1 Description statistique sommaire des séries : plages de variations, ordre de grandeur du rapport entre maxima et minima, moyenne, déviation standard et coefficient de variation.

Table 1 Succinct statistical description of the series: variation ranges, order of magnitude of the relationship between maximum and minimum, average, standard deviation and coefficient of variation.

\begin{tabular}{|c|c|c|c|c|c|c|c|}
\hline \multirow{2}{*}{ Descripteurs } & \multirow{2}{*}{ Unités } & \multicolumn{2}{|c|}{ Étendue } & \multirow{2}{*}{$\begin{array}{l}\text { Max } \\
/ \text { Min }\end{array}$} & \multirow{2}{*}{$\bar{X}$} & \multirow{2}{*}{ STD } & \multirow{2}{*}{$\begin{array}{l}\text { CV } \\
(\%)\end{array}$} \\
\hline & & Min & Max, & & & & \\
\hline \multicolumn{8}{|l|}{$\begin{array}{l}\text { ENVIRONNEMENT } \\
\text { (strate } 2-13 \mathrm{~m} \text { ) }\end{array}$} \\
\hline Température & ${ }^{\circ} \mathrm{C}$ & 8,8 & 9,7 & - & 9,3 & 0,2 & 1,9 \\
\hline $\begin{array}{l}\text { Stabilité verticale } \\
\text { (fréq. de Brünt-Vaïsälä) }\end{array}$ & $\mathrm{s}^{-2}$ & 0,35 & 0,47 & 1,4 & 0,43 & 0,04 & 8,9 \\
\hline Transparence & $\%$ & 15,1 & 34,3 & 2,3 & 30,4 & 3,0 & 9,8 \\
\hline $\mathrm{pH}$ & & 8,2 & 8,3 & 1,0 & 8,2 & 0,03 & 0,4 \\
\hline $\mathrm{O}_{2}$ dissous & $\%$ & 132 & 144 & 1,1 & 138 & 2,9 & 2,1 \\
\hline \multicolumn{8}{|c|}{ BIOMASSE (strate 0-20 m) } \\
\hline Cyanobactéries & $\mathrm{g} \mathrm{m}^{-2}$ & 14 & 38 & 2,7 & 25 & 5,72 & 23 \\
\hline Zooplancton & $m g m^{-2}$ & 13 & 219 & 17 & 90 & 0,06 & 61 \\
\hline Rapport Cyano. /Zoopl. & - & 130 & 1180 & 9 & 378 & 230 & 61 \\
\hline
\end{tabular}




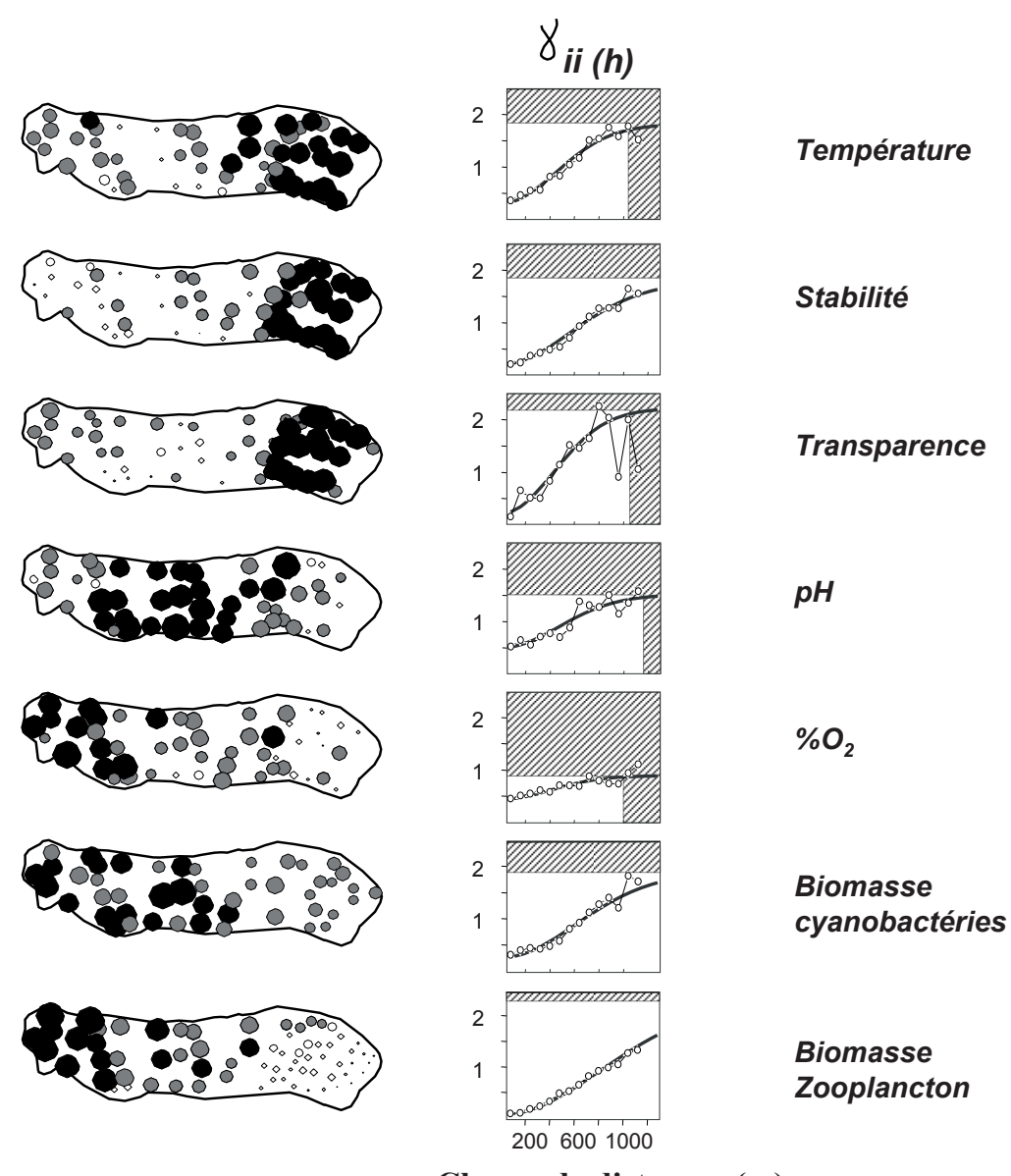

Classes de distances (m)

Figure 2 Répartitions horizontales des variables physico-chimiques relevées à la sonde multiparamètres (strate 2-13 m), des biomasses (strate 0-20 m) et variogrammes correspondants. Sur les cartes, le minimum minimorum est repéré par un losange; les 3 classes de valeurs distinguées par les symboles clairs, grisés et noirs ont respectivement pour bornes inférieures $1 \%, 40 \%$ et $80 \%$ de l'étendue de chaque variable. Sur les graphiques de droite, les variogrammes expérimentaux sont en traits fins et les variogrammes théoriques ajustés en trait gras. Les aires hachurées indiquent les limites du palier et de la portée.

Horizontal distributions of the physico-chemical variables obtained with the multiparameter probe (layer 2-12 m), biomass (layer 0-20 m) and corresponding variograms. On the charts, the minimorum minimum is located by a rhombus; the 3 classes of values indicated by the clear symbols, greyed and black, respectively for lower limits 1\%, $40 \%$ and $80 \%$ of the extension from each variable. On the graphs on the right-hand side, the experimental variograms are in fine lines and the theoretical adjusted variograms in bold lines. The hatched surfaces indicate the limits of the sill and the range.

\subsection{Structures spatiales déduites des variogrammes}

Les variogrammes expérimentaux sont très lisses pour le zooplancton et l'oxygène dissous, et plus rugueux pour la température, la stabilité et les cyanobactéries (Figure 2). Pour la transparence et le $\mathrm{pH}$, les variogrammes sont très rugueux (Figure 2). Une croissance d'abord lente de la variance avec la distance inter-station (soit une lente dégradation aux petites échelles spatiales de l'information) caractérise indifféremment les variables biologiques et environnementales. Ces variables sont également caractérisées par l'existence d'une portée (indépendance des observations, et donc forte dégradation de l'information d'une station quand on s'en éloigned'une distance allant de 1000 à $2000 \mathrm{~m}$ selon les variables considérées). La biomasse zooplanctonique globale se distingue par une longue portée et par un ajustement avec un modèle puissance, caractéristique de variables très régulières. Pour les autres variables, c'est le modèle gaussien qui s'ajuste le mieux aux variogrammes expérimentaux. La valeur de la portée permet de distinguer trois ensembles de variables :

1) les variables environnementales à l'exclusion de la stabilité, de portées analogues $(1000-1150 \mathrm{~m})$;

2) la stabilité et les cyanobactéries à plus longue portée (1 400-1 $500 \mathrm{~m}$ ); 
$\mathrm{Nb}$ de couples

150

50

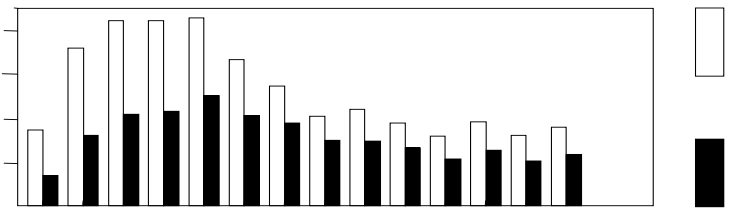

\section{1 stations}

54 stations
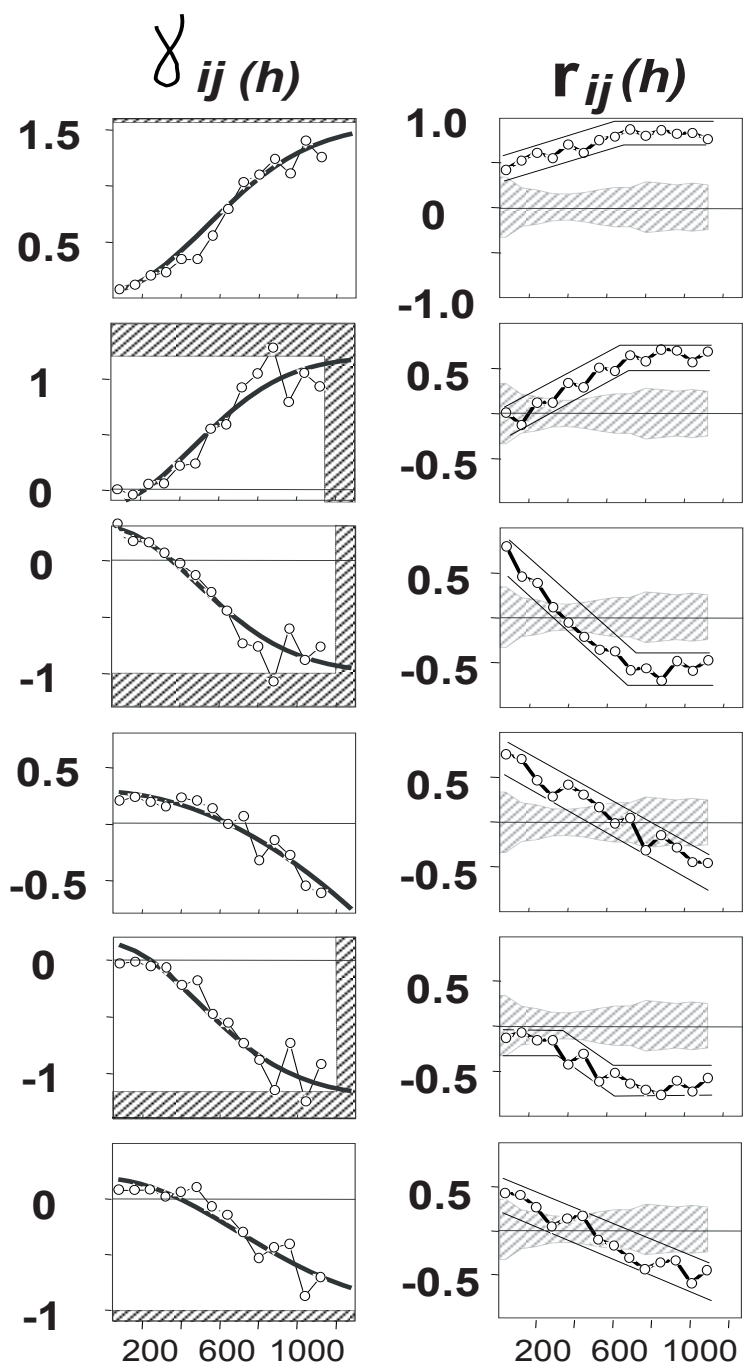

\author{
Stabilité
}

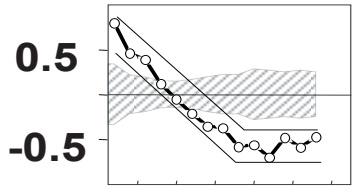

pH

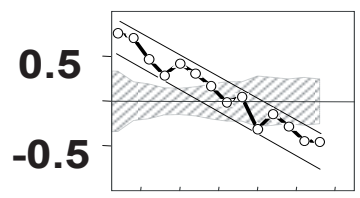

$\% \mathrm{O}_{2}$

Transparence

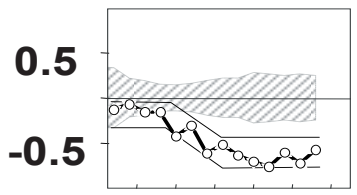

Biomasse

cyanobactéries

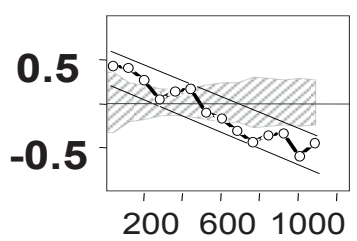

Biomasse

Zooplancton

\section{Classes de distances (m)}

Figure 3 Évolution de la relation existant entre la température et les variables biotiques et abiotiques. Graphiques de gauche : variogrammes croisés expérimentaux $\left(\gamma_{\mathrm{ij}}(\mathrm{h})\right)$ (trait fin) et théoriques (trait gras). Le nombre de couples de valeurs obtenus pour chaque classe de distance est indiqué sur l'histogramme du haut (71 pour les paramètres biologiques, 54 pour les paramètres physicochimiques). Les aires hachurées indiquent les limites du palier et de la portée. Graphiques de droite : coefficients de codispersion spatiale $\left(r_{i j}(h)\right)$. La tendance de la liaison avec la température est soulignée par des traits fins. À titre indicatif, le seuil de significativité $(\alpha=0,05)$ du coefficient de corrélation linéaire correspondant au nombre de paires de valeurs de chaque classe de distance est indiqué par des hachures.

Evolution of the relation between the temperature and the biotic and abiotic variables. Left-hand graphs: experimental variograms $\left(\gamma_{i j}(b)\right)$ (fine line) and theoretical (bold line). The number of couples of values obtained for each class of distance is indicated on the top histogram (71 for biological parameters, 54 for physico-chemical parameters). The hatched surfaces indicate the limits of the sill and the range. Right-hand graphs: coefficients of co-dispersion $\left(r_{i j}(b)\right)$. The trend of the connection with the temperature is underlined by fine lines. As an indication, the significance threshold $(a=0.05)$ of the linear coefficient of correlation corresponding to the number of pairs of values of each class of distance is indicated by hatchings. 
3) la biomasse zooplanctonique globale sans portée, en regard de la taille du lac. Le zooplancton global se distingue aussi de celui des autres variables par un très faible effet de pépite ( $4 \%$ du palier). Ce paramètre prend des valeurs beaucoup plus fortes pour la température et les cyanobactéries (20 à $18 \%$ du palier), et très fortes pour le $\mathrm{pH}$ et l'oxygène dissous (50 à $100 \%$ ).

\subsection{Covariations}

Le couple T-Stabilité se distingue des autres couples (Figure 3) :

1) par une covariation positive à toutes les échelles;

2) par les plus fortes valeurs absolues enregistrées pour le coefficient de codispersion spatiale $\left(\mathrm{r}_{\mathrm{ij}}(\mathrm{h})>0,80\right.$ pour $\mathrm{h}>550-600 \mathrm{~m}$ );

3) par la plus faible amplitude absolue de variation de celui-ci. Cette forte liaison entre température et stabilité thermique est des plus prévisible mais les graphes indiquent que la relation bi-variée se modifie avec l'échelle spatiale et se renforcent lorsqu'on passe des petites aux moyennes échelles $\left(\mathrm{r}_{\mathrm{ij}}(\mathrm{h})\right.$ passant de 0,43 à 0,88 pour $\left.80<\mathrm{h}<650 \mathrm{~m}\right)$.

Le couple T-Transparence se distingue du précédent par une plus forte amplitude de variation de $\mathrm{r}_{\mathrm{ij}}(\mathrm{h})(0,84$ unités pour $80<\mathrm{h}<880 \mathrm{~m})$. Au signe près, les graphes des couples T-Transparence et T-Cyanobactéries présentent un comportement analogue. L'amplitude de variation de $\mathrm{r}_{\mathrm{ij}}(\mathrm{h})$ est toutefois plus accusée pour ce dernier $(-0,06$ à $-0,76)$.

Pour les couples $\mathrm{T}-\mathrm{pH}$ et $\mathrm{T}-\mathrm{O}_{2}$, les covariations sont négatives aux petites échelles et positives aux grandes. De même, mais à un degré moindre, le cas s'observe pour T-Métazoaires. Les premiers se distinguent par une très forte modification spatiale de la liaison (amplitude de variation de $\mathrm{r}_{\mathrm{ij}}(\mathrm{h})$ respectivement de 1,50 et 1,20 pour le $\mathrm{pH}$ et l'oxygène; 0,70 seulement pour les métazoaires). Cette dérive de la corrélation des incréments se développe sur toute la plage d'échelles explorées dans le cas de l'oxygène (également, semblerait-il, dans le cas moins typé $\mathrm{du}$ zooplancton). Pour le $\mathrm{pH}$, le coefficient de codispersion se stabilise par contre vers $700<\mathrm{h}<800 \mathrm{~m}$ ).

\section{DISCUSSION}

Étendue des séries: Comparativement aux données de la littérature (Tableau 2), et bien que nos observations aient été faites au début des successions saisonnières, l'amplitude de variation horizontale observée pour la biomasse zooplanctonique est assez forte. Cette biomasse, parallèlement aux gradients verticaux, n'atteint en effet son maximum qu'en juin-juillet dans le lac de Nantua (FEUILLADE, 1985). La campagne a été effectuée en période de reproduction du gardon (Rutilus rutilus), espèce dominant le peuplement ichtyaire (STICKELREISSER, 1990). À cette période, la prédation par le poisson était donc encore faible. Or, la variabilité spatiale du zooplancton lacustre augmente avec les effectifs (DOWNING

Tableau 2 Plages de variations horizontales des effectifs totaux du zooplancton crustacéen total rapportées pour différents lacs.

Table 2 Coefficient of variation of the total number of zooplankton for different lakes.

\begin{tabular}{|c|c|c|c|c|c|}
\hline Lac & $\begin{array}{c}\text { Superficie } \\
\left(\mathrm{km}^{2}\right)\end{array}$ & $\begin{array}{c}\text { Nb. de } \\
\text { stations }\end{array}$ & Saison & CV $(\%)$ & Auteurs \\
\hline \multirow[t]{5}{*}{ Winnipeg (Canada) } & 23750 & $36^{(1)}$ & juin & $16-17^{(2)}$ & Patalas et Salki, 1992 \\
\hline & & $51^{(1)}$ & juillet & $8-17^{(2)}$ & \\
\hline & & $49^{(1)}$ & juil.-août & $23-9^{(2)}$ & \\
\hline & & $37^{(1)}$ & sept. & $36-11^{(2)}$ & \\
\hline & & $49^{(1)}$ & octobre & $43-28^{(2)}$ & \\
\hline Pyhäselkä (Finlande) & 263 & 33 & mai & 34 & Viljanen et Karjalainen, 1993 \\
\hline \multirow[t]{3}{*}{ Paasivesi (Finlande) } & 101 & 34 & août & 49 & Viljanen et Karjalainen, 1993 \\
\hline & & & juillet & 51 & \\
\hline & & & sept. & 24 & \\
\hline Léman (France- & 582 & $21^{(3)}$ & avril & 50 & Angeli et al., 1991 \\
\hline Suisse) & & $33^{(4)}$ & avril & 52 & Pinnel-Alloul et al. (1999) \\
\hline Nantua (France) & 1,4 & 71 & mai & 61 & cet article \\
\hline
\end{tabular}


et al., 1987; PINEL-ALLOUL et al., 1988), le développement des structures thermiques et celui de la pression de prédation (BOIKOVA, 1986; GLIWICZ et RYBOWSKA, 1992; LAIR et al., 1993; URABE, 1990). En revanche, les forts gradients verticaux de la fin du printemps à l'automne, et la tendance que présente une majorité de métazoaires planctoniques à se concentrer au-dessus de la couche d'Oscillaires, sont des facteurs qui amplifient l'impact de l'advection et le développement de fortes concentrations ou raréfactions locales et transitoires d'organismes (GEORGE et EDWARDS, 1973, 1978).

Structures spatiales et covariations: la quantification de l'hétérogénéité spatiale du zooplancton lacustre s'est longtemps fondée sur des indices globaux de dispersion (DOWNING, 1991), éventuellement sur l'analyse de variance (PINELALLOUL et al.,1988), mais plus rarement sur des méthodes restituant les grands traits structuraux (PINEL-ALLOUL et PONT, 1991; PINEL-ALLOUL et al., 1999). Les possibilités de comparaison sont donc assez limitées. Pour une plage très différente d'échelles d'observation ( $3 \mathrm{~km}-70 \mathrm{~km}$ ), les modèles gaussien et puissance caractérisent également les variables physico-chimiques et biologiques explorées dans le lac Léman à une étape également précoce des successions (PINELALLOUL et al., 1999). En saison chaude, à petite échelle $(10 \mathrm{~m}-150 \mathrm{~m})$, et pour les crustacés d'un petit lac dystrophe où la prédation par les invertébrés est forte en été, PINELALLOUL et PONT (1991) ont au contraire observé, pour un prédateur crustacéen et sa proie, un modèle de répartition en " trous et taches » avec alternance des essaims de proies et de ceux du prédateur. Il apparaît à ce niveau très global d'analyse, que les processus impliqués dans le développement des structures n'affectent les variables enregistrées ni avec la même force, ni aux même échelles. On remarque ainsi que les variables reflétant l'activité métabolique de la masse d'eau $(\mathrm{pH}$, $\mathrm{O}_{2}$ ) présentent une faible structuration spatiale aux échelles explorées et un énorme effet de pépite, suggérant qu'une partie notable de la variance totale de l'activité métabolique s'exprimait à une échelle inférieure à la maille d'échantillonnage $(<100 \mathrm{~m})$. Ainsi, toutes les variables covarient fortement avec la température à l'exception de la stabilité. Cette dépendance des variables biotiques et abiotiques avec les structures thermiques reflétant les mouvements de l'eau, se modifie fortement avec l'échelle d'observation. Avec l'augmentation de la distance inter-stations, elle passe de fortes valeurs positives à de fortes valeurs négatives pour les descripteurs dépendants d'activité métabolique globale ( $\mathrm{pH}$ et $\mathrm{O}_{2}$ ) et dans une moindre mesure, pour le zooplancton. Pour les cyanobactéries, qui représentent pourtant l'essentiel de la biomasse, la corrélation est également assez fortement négative, au-delà de $\mathrm{h}>500 \mathrm{~m}$, mais s'annule par contre à petite échelle.

Implications pratiques pour les évaluations quantitatives: compte tenu des faibles amplitudes de variations des descripteurs physico-chimiques relevées à cette époque de l'année, ces structures auraient peu d'incidence sur l'erreur commise dans le cadre d'un suivi effectué à partir d'une perception très locale de l'écosystème. Au niveau du plancton, si les amplitudes de variations sont fortes, elles se rapportent toutefois à un gradient amont-aval assez régulier, et donc au cas de figure le moins défavorable pour des estimés fondés en routine sur la région centrale du lac. À titre indicatif, nous avons calculé l'erreur d'évaluation commise à partir de mesures faites aux deux stations les plus profondes. Le jour de la campagne, le taux d'erreur calculé par la formule $[\max (\mathrm{Zi}$, $\left.\mathrm{Zm}) / \min (\mathrm{Zi}, \mathrm{Zm})^{*} 100\right]$ aurait été de 230 et $374 \%$ pour les cyanobactéries, et de 140 et $170 \%$ pour le zooplancton. Un cas de figure considérablement plus défavorable peut par contre se présenter quand régions pélagiques ou littorales servent de refuge vis-à-vis de la prédation par les alevins, et que se développent et se maintiennent durant la saison chaude de forts gradients de biomasse et de structure de peuplement entre régions pélagique et littorale. Pour des crustacés, BOIKOVA (1986) constatait ainsi durant l'été des différences rive-large d'effectifs excédant deux ordres de grandeur.

En conclusion, bien que l'exploration se soit déroulée tôt en saison (faible développement des structures thermiques; faible amplitude de variation des descripteurs environnementaux), la biomasse zooplanctonique globale présentait à la fois une forte structuration spatiale et une assez forte amplitude de variations horizontales. Il en va de même du rapport oscillaires: métazoaires, bien que les biomasses de ces deux catégories planctoniques aient présenté des gradients longitudinaux assez réguliers et de même sens. Il est donc à craindre que les phénomènes d'advection, qui semblent avoir dominé la répartition à grande échelle des biomasses planctoniques, soient associés à des variations notables, à grande échelle, de la structure du peuplement. À l'époque de la campagne, contrairement à ce qui se passe pour les oscillaires et pour une partie des descripteurs environnementaux, la dégradation de l'information obtenue dans des conditions locales n'était pas très rapide pour la biomasse zooplanctonique globale. Pour ce descripteur, nous n'avons pas non plus constaté d'effet de pépite important à la différence de ce qui a été observé pour les variables reflétant l'activité métabolique globale et pour lesquelles les structures horizontales semblent avoir été exprimées à une échelle inférieure à $100 \mathrm{~m}$. Enfin, sur la plage d'échelles analysées (100 - $1250 \mathrm{~m}$ ), la relation entre température et variables dépendantes, tant physicochimiques que biologiques, varie considérablement avec la distance inter-station. Au total, cette approche fondée sur des variables globales souligne la difficulté d'extrapoler les liaisons entre variables ou processus à d'autres échelles que celle(s) observée(s). Il serait nécessaire, lorsque les conditions tant matérielles que temporelles et météorologiques sont réunies, de réaliser un repérage préalable léger (AMNANIEU et al., 1989; AVOIS et al., 2000) des structures exprimées avant de définir un protocole de suivi pour chaque catégorie d'éléments du système. Les structures spatiales et temporelles 
des biocénoses jouant un rôle central dans le fonctionnement des écosystèmes.

\section{RÉFÉRENCES BIBLIOGRAPHIQUES}

AMANIEU M., LEGENDRE P., TROUSSELLIER M. et G.-F. FRISONI, 1989. Le programme Écothau : théorie écologique et base de la modélisation. Oceanol. Acta, 12, 189-199.

ANGELI A., DUFOUR P., GERDEAUX D., GUILLARD J. et J.-P. PELLETIER, 1991. Variabilité horizontale des sels nutritifs et des biomasses planctoniques dans le Léman : incidence de la bise en période de faible stabilité. Dans: Hommage à F.-A. Forel: Troisième conférence internationale des limnologues d'expression française. VERNET, J.-P. (éditeur), Morges, 219-222.

AVOIS C., LEGENDRE P. , MASSON S. et B. PINELALLOUL, 2000. Is the sampling strategy interfering with the study of spatial variability of zooplankton communities? Can. J. Fish. Aquat. Sci., 57, 1940-1956.

ARMSTRONG M., RENARD D., RIVOIRARD J. et P. PETITGAS, 1992. Geostatistics for fish survey data. ICES, Centre de géostatistique, Fontainebleau, France, 90 p.

BOIKOVA O.S., 1986. Horizontal distribution of crustaceans in Lake Globokoe. Hydrobiol., 141, 113-123.

BÜRGI H.R., ELSER J.J., RICHARDS R.C. et C.R. GOLDMAN, 1993. Zooplankton patchiness in Lake Tahoe and Castle Lake USA. Verh. Internat. Verein. Limnol., 25, 378-382.

DIRNBERG J.M. et S.T. THRELKELD, 1986. Advective effects of a reservoir flood on zooplankton abundance and dispersion. Freshwater Biol., 16, 387-396.

DOWNING J.A., 1991. Biological heterogeneity in aquatic ecosystems. Dans: Kolasa J. \& S.T.A. PICKETT (éditeurs). Ecological heterogeneity, 160-180.

DOWNING J.A., PERUSSE M. et Y. FRENETTE, 1987. Effect of interreplicate variance on zooplankton sampling design and data analysis. Limnol. Oceanogr., 32, 673-680.

DUTILLEUL P., 1993. Spatial heterogeneity and the design of ecological field experiments. Ecol., 74, 1646-1658.

DUTILLEUL P. et P. LEGENDRE, 1993. Spatial heterogeneity against heteroscedasticity: an ecological paradigm versus a statistical concept. Oiko., 66, 152-171.
FEUILLADE, J. (Éditeur), 1985. Caractérisation et essais de restauration d'un écosystème dégradé : le lac de Nantua, $165 \mathrm{p}$.

FEUILLADE M. et J.C. DRUART, 1994. The long-term effect of the sewage diversion on the phytoplankton composition and biomass. Arch. hydrobiol. beih., 41, 55-76.

GEORGE D.G. et R.W. EDWARDS, 1973. Daphnia distribution within Langmuir circulations. Limnol. Oceanogr., 18, 798-800.

GEORGE D.G. et R.W. EDWARDS, 1978. The effect of wind on the factors influencing the saptial distribution of chlorophyll $a$ and crustacean plankton in a shallow eutrophic recervoir. J. Appl. Ecol., 13, 667-690.

GELLER W., PINTO-COELHO R. et H.R. PAULI, 1992. The vertical distribution of zooplankton (Crustacea, Rotatoria, Ciliata) and their grazing over the diurnal and seasonal cycles in Lake Constance. Arch. Hydrobiol. Beilh. Ergebn. Limnol., 35, 79-85.

GLIWICZ Z. et M. RYBOWSKA, 1992. Shore avoidance in zooplankton: a predator-induced behavior or predatorinduced mortality. J. Plankton Res., 14, 1331-1342.

GOOVAERTS P., 1992. Factorial kriging analysis: a useful tool for exploring the structure of multivariate soil inforamtion. J. Soil. Sci., 43, 597-619.

HANAZATO T., 1992. Direct and indirect effects of lowoxygen layers on lake zooplankton communities. Arch. Hydrobiol. Beilh. Ergebn. Limnol., 35, 87-98.

HART R.C., 1978. Horizontal distribution of the copepod Pseudodiaptomus hessei in subtropical Lake Sibaya. Freshwater Biol., 8, 415-421.

IMBODEN D.M., EID B.S.F., JOLLER T., SCHURTER M. et J. WETZEL, 1979. MELIMEX, an experimental heavy metal pollution study: vertical mixing in large limnocorral. Schweiz. Z. Hydrol., 41, 2, 177-183.

JOHANNSSON O.E., MILLS E.L. et R. O'GORMAN, 1991. Changes in the nearshore and offshore zooplankton communities in Lake Ontario: 1981-88. Can. J. Fish. Aquat. Sci., 48, 1546-1557.

JOURNEL A. et C. HUIJBREGTS, 1978. Mining geostatistics. Academic press, London.

KALIKHMAN I., WALLINE P. et M. GOPHEN, 1992. Simultaneous patterns of temperature, oxygen, 
zooplankton and fish distribution in Lake Kinneret, Israel. Freshwater Biol., 28, 337-347.

LACROIX G. et F. LESCHER-MOUTOUÉ, 1995. Spatial patterns of planktonic microcrustaceans in a small shallow lake. Hydrobiologia, 300, 205-217.

LAIR N., TALEB H. et P. REYES-MARCHANT, 1993. Zooplankton diversity response to predation pressure in littoral and limnetic zones of a eutrophic lake, in France. Verh. Int. Verein. Limnol., 25, 603-607.

LEGENDRE P., 1993. Spatial autocorrelation: Trouble or new paradigm? Ecol., 74, 16591673.

LEGENDRE P. et M.J. FORTIN, 1989. Spatial pattern and ecological analysis. Vegetatio, 80, 107138.

LEMMIN U., 1995. Limnologie physique. Chap. 2. Dans : Limnologie générale. POURRIOT R. et MEYBECK M. (Éditeurs), 60-114.

MASSON S., PINEL-ALLOUL B. et P. DUTILLEUL, 2004. Spatial heterogeneity of zooplankton biomass and size structure in southern Quebec lakes: variation among lakes and within lakeamong epi-, meta- and hypolimnion strata. J. Plankton Res., 26, 12, 1441-1458.

MATHERON G., 1971. The theory of regionalized variables and their applications. Les cahiers du centre de morphologie mathématique, fasc. 5, Centre de géostatistique, Fontainebleau, France.

PATALAS K. et A. SALKI, 1992. Crustacean plankton in Lake Winnipeg: variation in space and time as a function of lake morphometry, geology, and climate. Can. J. Fish. Aquat. Sci., 49, 1035-1059.

PETITGAS P. et T. LAFONT, (1997). EVA2 : Estimation of variance, version 2. A geostatistical software package on Windows 95 for estimating precision of fish strock assessment surveys. ICES CM, 1997/Y 22.

PINEL-ALLOUL B., GUAY C., ANGELI N., LEGENDRE P., DUTILLEUL P., BALVAY G., GERDEAUX D. et J. GUILLARD, 1999. Large-scale spatial heterogeneity of macrozooplankton in Lake of Geneva. Can. J. Fish. Aquat. Sci., 56, 1437-1451.

PINEL-ALLOUL B., DOWNING J.A., PERUSSE M. et G. GODIN-BLUMER, 1988. Spatial heterogeneity in freshwater zooplankton: variations with body size, depth and scale. Ecol., 69, 1393-1400.
PINEL-ALLOUL B. et D. PONT, 1991. Spatial distribution patterns in freshwater macrozooplankton: Variation with sampling scales. Can. J. Zool., 69, 1557-1570.

PETITGAS P. et T. LAFONT, 1997. EVA2: Estimation of variance, version 2. A geostatistical software package on Windows 95 for estimating precision of fish stock assessment surveys. ICES CM,1997/Y 22.

REYNOLDS C.S., 1984. The ecology of freshwater phytoplankton. Cambridge University Press, Cambridge, U.K.

SOMMER U., 1989. Plankton ecology: Succession in plankton communities. Brock/Springer series. Dans : Contemporary Bioscience. Springler-Verlag.

STICKELREISSER V., 1990 Echosondage - echointégration : Acquisition et traitement des données sur le lac de Nantua en mai 1990. Rapp. I.L., 02-90, 35 p.

URABE J., 1990. Relative importance of temporal and spatial heterogeneity in the zooplankton community of an artificial reservoir. Hydrobiol., 184, 1-6.

VILJANEN M. et J. KARJALAINEN, 1993. Horizontal distribution of zooplankton in two large lakes in Eastern Finland. Verh. Internat. Verein. Limnol. 25, 548-551.

WACKERNAGEL H., 1988. Geostatistical technics for interpreting multivariate spatial information. Dans : C.F. Chung et al. (Éditeurs), Quantitative analysis of mineral and energy resources. Reidel, Dordrecht, pp. 393-409.

WEIDER L.J. et H.B. STICH, 1992. Spatial and temporal heterogeneity of Daphnia in Lake Constance; intra- and interspecific comparisons. Limnol. Oceanogr., 37, 6, 1327-1334.

YATES S.R, YATES M.V. et D.M. WALTERS, 1989. Geostatistics for waste management : a user's manual for the GEOPACK (version 1.0) geostatistical sotfware system. $70 \mathrm{p}$. 